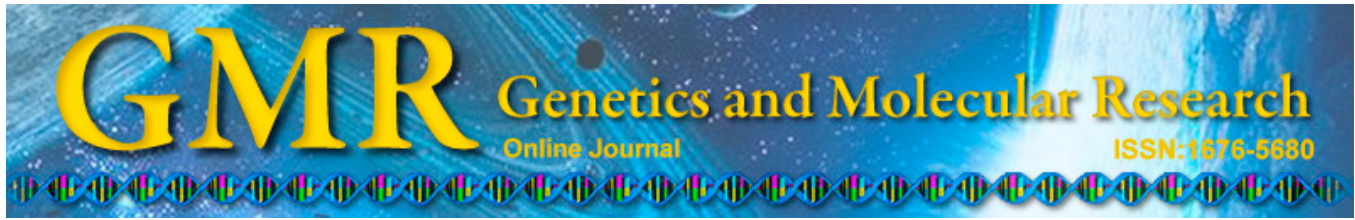

\title{
Sublingual vein parameters, AFP, AFP-L3, and GP73 in patients with hepatocellular carcinoma
}

\author{
J. Zhao ${ }^{1,2}$, L.-Y. Guo ${ }^{1}$, J.-M. Yang ${ }^{1}$ and J.-W. Jia ${ }^{1}$ \\ ${ }^{1}$ Tianjin Second People's Hospital, Tianjin, China \\ ${ }^{2}$ Tianjin Institute of Hepatology, Tianjin, China \\ Corresponding author: J.-W. Jia \\ E-mail: zhaojvvip@163.com
}

Genet. Mol. Res. 14 (2): $7062-7067$ (2015)

Received November 4, 2014

Accepted February 10, 2015

Published June 26, 2015

DOI http://dx.doi.org/10.4238/2015.June.26.16

ABSTRACT. This study evaluated the diagnostic value of alphafetoprotein (AFP), AFP heterogeneity 3 (AFP-L3), Golgi protein 73 (GP73), and sublingual vein parameters in hepatocellular carcinoma (HCC). Levels of serum AFP, AFP-L3, GP73, and sublingual vein scores were measured in 34 patients with chronic hepatitis, 65 patients with post-hepatitis B cirrhosis, 71 patients with $\mathrm{HCC}$, and 6 healthy controls. Logistic regression analysis was used to explore potential correlations. Sublingual vein grades in patients with HCC were higher than those in the other three groups; sublingual vein scores were also different between groups; combined diagnosis using AFP, GP73, and sublingual vein grade was superior to the individual parameters alone or when only two were used in different combinations. Thus, sublingual vein grade can be considered as an independent risk factor for diagnosis of HCC. Furthermore, combined detection with AFP, GP73, and sublingual vein grade is simple, inexpensive, and effective. It may therefore be suitable for screening high-risk populations for early diagnosis of HCC.

Key words: Sublingual vein; Hepatocellular carcinoma; Alpha-fetoprotein; AFP heterogeneity 3; Golgi protein 73 


\section{INTRODUCTION}

Hepatocellular carcinoma (HCC) has a high prevalence, accounting for $4 \%$ of all newonset malignancies annually, with an increasing incidence worldwide (Yao, 2004). Although surgical resection remains the most effective method for treatment of HCC, many patients are not candidates for surgical treatment. A period of approximately 10 months passes from an observable increase in alpha-fetoprotein (AFP) to the establishment of subclinical HCC, suggesting that early diagnosis is feasible (Wang and Zhang, 2003). Many tumor markers are currently used to improve the sensitivity and specificity of diagnosis. In this study, AFP, AFP heterogeneity 3 (AFP-L3), golgi protein 73 (GP73), and sublingual vein parameters were investigated (Kladney et al., 2002; Block et al., 2005; Marrero et al., 2005) in order to explore their value in early diagnosis of HCC.

\section{MATERIAL AND METHODS}

\section{Clinical data}

All patients were hospitalized or they were outpatients at the Second People's Hospital of Tianjin between October 2008 and November 2010. The study included 170 subjects, including 34 patients with chronic hepatitis B, 65 patients with post-hepatitis B cirrhosis, and 71 patients with HCC; 6 healthcare workers at the Second People's Hospital of Tianjin were selected as healthy controls during the same period. There were 120 men and 56 women with a mean age of $48.3 \pm 15.5$ years (range 20-80 years). Patients in the chronic hepatitis, cirrhosis, and HCC groups were all undergoing conventional and symptomatic treatment. There were no significant differences in age or sex between groups $(\mathrm{P}>0.05)$.

\section{Sublingual vein parameters and scoring}

Trunk and branch morphology, line feed, diameter, length, color of veins, presence of hyperemia, engorgement, congestion spots, and congestion streaks in tongue ventral fine vasculature were assessed. The scoring criteria of the tongue and pulse are reported in "Basic TCM for Cancer Diagnosis".

\section{Serum samples and evaluation}

Venous blood $(5 \mathrm{~mL})$ from fasting subjects was drawn once in a standard blood collection tube. Serum was rapidly separated $(3000 \mathrm{rpm}, 1.5 \mathrm{~min})$ and stored at $-20^{\circ} \mathrm{C}$ for $<3$ months before testing. AFP and AFP-L3 were detected using electrochemiluminescence; GP73 was tested by enzyme-linked immunosorbent assay. The reference ranges used to determine positivity were as follows: AFP: non-HCC (negative) $<200 \mathrm{ng} / \mathrm{mL}$; HCC (positive) $\geq 200 \mathrm{ng} / \mathrm{mL}$; AFP-L3: positive when $\geq 10 \%$ of total AFP; GP73: non-HCC (negative) $<150 \mathrm{ng} / \mathrm{mL}$; HCC (positive) $\geq 150 \mathrm{ng} / \mathrm{mL}$.

\section{Statistical analysis}

SPSS ver. 11.5 (Chicago, IL, USA) was used for all statistical analyses; independent samples were compared using the Wilcoxon test; diagnosis of HCC and multivariate relation- 
ships were tested by logistic regression analysis. A P $<0.05$ was considered to be significant.

\section{RESULTS}

\section{Sublingual vein analysis}

The sublingual veins of 176 subjects were observed, scored, and graded ( 0 points as grade $1,1-5$ as grade 2, 6-9 as grade 3 , and $\geq 10$ as grade 4 ). The results are shown in Table 1. A rank sum test of multiple independent samples was conducted that showed significant differences between groups $(\mathrm{P}<0.05)$; pairwise comparisons between groups also showed significant differences $(\mathrm{P}<0.05)$. Moreover, sublingual vein scores between the HCC and non-HCC groups were significantly different $(\mathrm{P}<0.05)$.

Table 1. Distribution of sublingual grades in the HCC, cirrhotic, hepatitis, and normal control groups.

\begin{tabular}{lcccc}
\hline Group (case) & \multicolumn{4}{c}{ Grade of sublingual veins } \\
\cline { 2 - 5 } & Grade 1 & Grade 2 & Grade 3 & Grade 4 \\
\hline HCC $(\mathrm{N}=71)$ & $23(13.07)$ & $10(5.68)$ & $6(3.41)$ & $32(18.18)$ \\
Cirrhosis $(\mathrm{N}=65)$ & $33(18.75)$ & $16(9.09)$ & $5(2.84)$ & $11(6.25)$ \\
Hepatitis $(\mathrm{N}=34)$ & $29(16.48)$ & $4(2.27)$ & $1(0.57)$ & $0(0.00)$ \\
Control $(\mathrm{N}=6)$ & $6(3.41)$ & $0(0.00)$ & $0(0.00)$ & $0(0.00)$ \\
Total & $91(51.70)$ & $30(17.05)$ & $12(6.82)$ & $43(24.43)$ \\
\hline
\end{tabular}

Impact of AFP, AFP-L3, and GP73 on sublingual vein scores

\section{Correlations between sublingual vein scores and levels of AFP and AFP-L3}

When considering an AFP $\geq 200 \mathrm{ng} / \mathrm{mL}$ and AFP-L3 $\geq 10 \%$ as positive criteria, there was no significant difference in sublingual vein scores between the AFP-positive and AFPnegative groups $(\mathrm{P}>0.05)$; in addition, there was no significant difference between the AFPL3 positive and AFP-L3 negative groups $(\mathrm{P}>0.05)$ (Table 2).

Table 2. Sublingual grades according to levels of AFP and AFP-L3.

\begin{tabular}{lcccc}
\hline & \multicolumn{3}{c}{ Grade of sublingual veins } \\
\cline { 2 - 5 } & Grade 1 & Grade 2 & Grade 3 & Grade 4 \\
\hline AFP (N) & 2 & 3 & 8 & 6 \\
$\geq 200 \mathrm{ng} / \mathrm{mL}$ & 56 & 47 & 35 & 19 \\
$<200 \mathrm{ng} / \mathrm{mL}$ & & 4 & 7 & 5 \\
AFP-L3 (N) & 1 & 49 & 38 & 18 \\
$\geq 10 \%$ & 54 & & \\
$<10 \%$ &
\end{tabular}

Between AFP groups, $\mathrm{P}=0.141$; between AFP-L3 groups, $\mathrm{P}=0.084$.

\section{Effect of GP73 on sublingual vein scores}

Spearman's rank correlation of the results of GP73 and sublingual vein scores showed no correlation between the two parameters $(\mathrm{P}>0.05)$ (Table 3$)$. 
Table 3. Spearman's rank correlation analysis of GP73 and sublingual vein scores.

\begin{tabular}{lcc}
\hline Spearman's rho & Sublingual vein score & GP73 \\
\hline Sublingual vein score & 1.000 & $0.135^{\mathrm{a}}$ \\
GP73 & $0.135^{\mathrm{a}}$ & 1.000 \\
\hline
\end{tabular}

${ }^{\mathrm{a}} \mathrm{P}=0.208$.

\section{Logistic regression analysis of the relationship of HCC diagnosis with AFP, AFP-L3, GP73, and sublingual vein scores}

Forward stepwise logistic regression analysis was used considering the results with AFP, AFP-L3, GP73, and sublingual vein scores as independent variables, with diagnosis of HCC as the outcome variable, 0.05 as the significance level of enrolled variables, and 0.10 as the significance level of removing variables (see equation belore). Three variables were entered into the regression equation, namely AFP, GP73, and sublingual vein score. In descending order, HCC diagnosis was influenced by GP73, sublingual vein score, and AFP; these variables were thus considered risk factors for diagnosis of HCC.

$$
\mathrm{P}=\frac{\exp \left(-2.857+0.006 \times \mathrm{AFP}+0.244 \times \mathrm{GP} 73^{\mathrm{c}}+0.124 \times \text { sublingual vein score }\right)}{1+\exp \left(-2.857+0.006 \times \mathrm{AFP}+0.244 \times \mathrm{GP} 73^{\mathrm{d}}+0.124 \times \text { sublingual vein score }\right)}
$$

where $A F P, G P 73$, and sublingual vein scores were all considered as detected values; after logarithmic conversion, GP73 met normal distribution criteria; outcome variables were assigned the diagnostic " 1 " and excluded "0"; $c$ : GP73; $d$ : GP73 was needed for the natural logarithm.

The area under the ROC curve of the combined use of AFP, GP73, and sublingual vein scores on $\mathrm{HCC}$ diagnosis was $0.845(\mathrm{P}=0.000)$ (Figure 1$)$; it was significantly greater than that of the individual parameters alone (AFP: 0.727; GP73: 0.675; sublingual vein score: 0.730) and in different pairwise combinations (AFP + GP73: 0.804; AFP + sublingual vein score: 0.805 ; GP73 + sublingual vein score: 0.771 ).

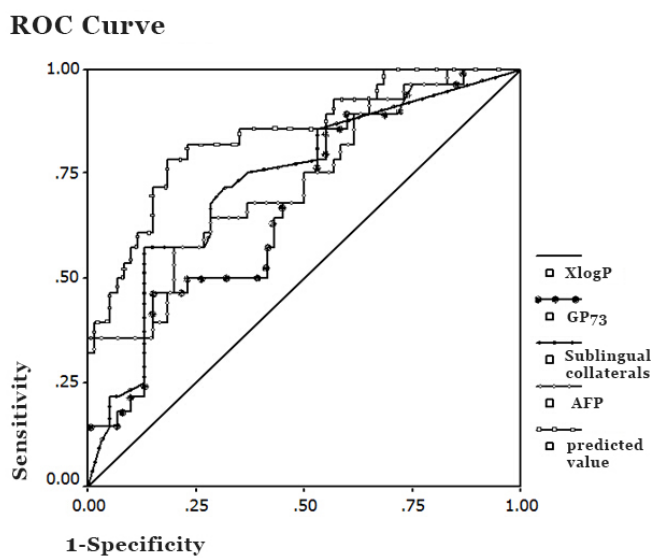

Figure 1. ROC curves of HCC detection and diagnosis by AFP, GP73, and sublingual scores and the combination of the three parameters. 


\section{DISCUSSION}

HCC is one of the most common malignancies. Many patients are not candidates for surgical resection and overall prognosis is poor (Wahab et al., 2014). Currently, AFP detection is combined with other imaging techniques such as ultrasonography, computed tomography, and magnetic resonance imaging to facilitate early clinical diagnosis of HCC, although the results are generally unsatisfactory. One of the major reasons for this is that although AFP plays an important role in the screening of HCC, owing to the commonly used serum reference range of 200-300 ng/mL, the rates of false positives and false negatives are high. Thus, AFP has limitations in making a distinction between HCC and benign liver disease. Given this, the use of serum AFP as a screening indicator for $\mathrm{HCC}$ has been questioned, and it is of interest to find additional markers for HCC.

In recent years, with the rapid development of gene technology, proteomics, tumor immunology, several potential tumor markers have been identified. These include AFP-L3, transforming growth factor $\beta 1$, vascular endothelial growth factor, GGT, and GP73, which are highly expressed in the serum of patients with HCC, and have attracted the most attention. Although these markers are not specific for HCC, they have high sensitivity and specificity in screening for cirrhosis and in diagnosis of $\mathrm{HCC}$ in specific populations. Indeed, they can complement the results of serum AFP and serve as composite markers of HCC to improve diagnosis. Detection methods for new markers and requirements for specimens vary widely, as do costs of the different tests. In the literature, GP73 and AFP-L3 have been reported to have high diagnostic sensitivity and specificity, respectively. The method used for their detection method is simple, economical, and easy to perform.

At the same time, the advantages of Chinese medicine in the early diagnosis of HCC should not be overlooked. Chinese medicine has a long history of interest in cancer, using such terms as Zhengjia, accumulation, stones, and so on. ShenJinao, of the Qing Dynasty, stated in "mechanisms and origins of miscellaneous diseases": although accumulation, Zhengjia, hernia, and lump are different, they can all be regarded as accumulation, because accumulation is the general term of stopping storage. In "Correction on the errors of medical works", it was stated, "belly-caking must be caused by blood agglomeration. While studying the mechanisms behind cancer, the blood stasis doctrine was recognized by many scholars, but because of difficulties in standardization and quantification, this did not lead to any significant breakthroughs. Many clinical and experimental studies have shown that the sublingual vein is closely related with blood stasis syndrome, and assessment of the sublingual vein has been demonstrated to be useful in diagnosis and prognosis of severe chronic liver disease, and can also be used as an ancillary indicator for blood stasis syndrome, as well as treatment and prognosis (Liu et al., 2003a,b; Gao et al., 2007). Previous investigations have also confirmed that there are significant differences in sublingual vein abnormalities between patients with HCC and those without. Thus, herein, the sublingual vein was considered as a representative sign of blood stasis syndrome, which was scored and graded to compare differences between the HCC and non-HCC groups.

Our study showed that sublingual vein scores were significantly higher in patients with HCC compared with the non-HCC groups, and that they were independently associated with HCC. Logistic regression analysis selected levels of AFP and GP73 as well as sublingual vein scores, which were then entered in an ROC analysis. This showed that the combined use of AFP, GP73, and sublingual vein scores for early detection and diagnosis of HCC was 
superior to the three parameters used individually or in a pairwise fashion. It further indicated that GP73 and thesublingual grade could be used as a strong complement to AFP. As these assays are easy to perform, interpret, and economical, they are suitable for following high-risk patients with HCC and for performing early screening of HCC.

\section{Conflicts of interest}

The authors declare no conflict of interest.

\section{REFERENCES}

Block TM, Comunale MA, Lowman M, Steel LF, et al. (2005). Use of targeted glycoproteins to identify glycoproteins that correlated with liver cancer in woodchucks and humans. Proc. Natl. Acad. Sci. U. S. A. 102: 779-784.

Gao JD, Yue XQ Liu Q, Yu H, et al. (2007). Clustering sublingual collaterals in patients with primary liver cancer research. J. Zhejiang Univ. Chin. Med. 31: 567-568.

Kladney RD, Cui X, Bulla GA, Burnt EM, et al. (2002). Expression of GP73, a resident Golgi membrane protein, in viral and nonviral liver disease. Hepatology 35: 1431-1440.

Liu Q, Yue XQ, Deng WZ, Wang Z, et al. (2003a). Application of integrated information of tongue diagnosis analysis system for primary liver cancer quantitative analysis of tongue color. Zhong Xi Yi Jie He XueBao 1: 180-183.

Liu Q, Yue XQ, Hou FG, Wang Z, et al. (2003b). Relationship between blood stasis and liver cancer sc - 1010 (this) related literature analysis. J. Shandong Univ. TCM 27:92-94.

Marrero JA, Romano PR, Nikolaeva O, Steel L, et al. (2005). Gp73, a resident Golgi glycol protein, is a novel serum marker for hepatocellular carcinoma. J. Hepatol. 43: 1007-1012.

Wahab MA, Shehta A, Hamed H, El Nakeeb A, et al. (2014) Predictors of recurrence in hepatitis C virus related hepatocellular carcinoma after hepatic resection: a retrospective cohort study. Eur. J. Med. 46: 36-41.

Wang BE and Zhang DF (2003). Modern hepatology. Science Press, Beijing, 851-885.

Yao GB (2004). Clinical hepatology. Shanghai Science and Technology Publishing House, Shanghai, 621-638. 